\title{
KSN heterozygosity is associated with continuous flowering of Rosa rugosa Purple branch
}

\author{
Mengjuan Bai', Jinyi Liu', Chunguo Fan', Yeqing Chen', Hui Chen', Jun Lu', Jingjing Sun', Guogui Ning ${ }^{2}$ and \\ Changquan Wang (1]
}

\begin{abstract}
Rose (Rosa spp.) plants flower via two contrasting methods: once flowering (OF) and continuous flowering (CF). Purple branch is a rare continuously flowering variety of Rosa rugosa that is extensively cultivated in China. However, the genetic basis of its CF behavior is unknown. We demonstrated that Purple branch is heterozygous for the TFL1 homolog KSN. One KSN allele with a $9 \mathrm{~kb}$ Copia insertion was found to be identical to that from continuously flowering Rosa chinensis Old blush. The other allele was found to be a functional wild-type allele. The overall expression of KSN was closely linked to the floral transition, and it was significantly repressed in continuously flowering Purple branch compared with OF Plena. The promoter region of the normal KSN allele was hypermethylated, and histone methylation at $\mathrm{H3H} 4, \mathrm{H} 3 \mathrm{~K} 9$, and $\mathrm{H} 3 \mathrm{~K} 27$ of the KSN gene locus was modified in continuously flowering Purple branch. Silencing of the DNA methyltransferase genes MET1 and CMT3 and the histone methyltransferase gene SUVR5 in Purple branch led to enhanced KSN expression, but silencing of the histone demethylase gene JMJ12 suppressed KSN expression. Therefore, the CF habit of Purple branch may be due to reduced expression of KSN caused by the halved dose and may be associated with epigenetic modifications together with retrotransposon insertions along the chromosome. Our study revealed a novel mechanism underlying the CF behavior of rose plants.
\end{abstract}

\section{Introduction}

Rosa rugosa Thunb., a member of the Rosaceae family, which is indigenous to Eastern Asia, was introduced into Europe and North America in the middle of the nineteenth century $^{1,2}$. In addition to producing fragrant flowers, this popular and economically valuable ornamental species has medicinal properties. $R$. rugosa flowers are widely used in traditional and folk medicine in China, Japan, and Korea due to the presence of secondary metabolites that exert pharmacological activities ${ }^{3}$. Essential oil from petals, known as "liquid gold", is a raw material used in perfumes, cosmetics, aromatherapy, spices, and the nutrition industry $^{4}$. Unlike modern roses (Rosa hybrida), which flower continuously throughout the year, most $R$. rugosa varieties

\footnotetext{
Correspondence: Changquan Wang (cqwang@njau.edu.cn)

${ }^{1}$ College of Horticulture, Nanjing Agricultural University, Nanjing 210095, China

${ }^{2}$ College of Horticulture and Forestry Sciences, Huazhong Agricultural

University, Wuhan 430070, China

These authors contributed equally: Mengjuan Bai, Jinyi Liu
}

flower once a year during the spring. Efforts to produce flowers year-round rely on screening and breeding new continuously flowering $R$. rugosa varieties.

Flowering, which involves the transition from vegetative growth to reproductive development, is controlled by external environmental cues and endogenous signals through six genetic pathways in Arabidopsis: the photoperiod, vernalization, autonomous, gibberellin, age, and temperature pathways ${ }^{5}$. Rose plants have three flowering methods: an once flowering (OF) period in the spring; continuous flowering (CF) during favorable growth seasons; and occasional reblooming, ${ }^{6,7}$.

Rose KSN, a homolog of TFL1 of Arabidopsis thaliana, acts as a flowering repressor to control OF/CF. In OF rose cultivars, the expression of $K S N$ is repressed in the winter and early spring, so the plants bloom only in late spring. After blooming, KSN expression is activated and represses further flower formation in the summer ${ }^{6,8,9}$. Continuously flowering Rosa chinensis Old blush contains a $9 \mathrm{~kb}$ Copia

\section{(c) The Author(s) 2021}

(c) (i) Open Access This article is licensed under a Creative Commons Attribution 4.0 International License, which permits use, sharing, adaptation, distribution and reproduction cc) in any medium or format, as long as you give appropriate credit to the original author(s) and the source, provide a link to the Creative Commons license, and indicate if changes were made. The images or other third party material in this article are included in the article's Creative Commons license, unless indicated otherwise in a credit line to the material. If material is not included in the article's Creative Commons license and your intended use is not permitted by statutory regulation or exceeds the permitted use, you will need to obtain permission directly from the copyright holder. To view a copy of this license, visit http://creativecommons.org/licenses/by/4.0/. 


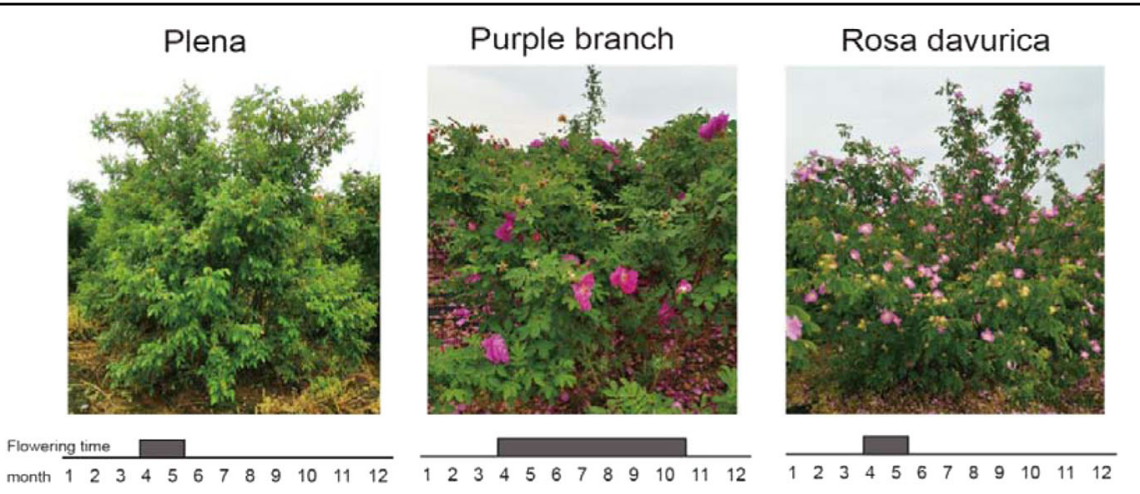

Fig. 1 Images of $\boldsymbol{R}$. rugosa Plena, $\boldsymbol{R}$. rugosa Purple branch and $\boldsymbol{R}$. davurica. R. rugosa Plena and R. davurica are OF species blooming from April to May. R. rugosa Purple branch is a continuously flowering variety that flowers from April to October. The flowering time is represented by the gray boxes

retrotransposon insertion in the second intron of $K S N$, and this insertion blocks KSN expression, enabling continuous flowering ${ }^{9}$. Old blush is hybridized with European rose plants to generate modern rose plants, in which the recurrent flowering trait has presumably been transferred into the modern rose plants ${ }^{6}$. Therefore, most continuously flowering rose cultivars are expected to harbor this mutated allele of KSN from Old blush. However, the presence of a null allele has been suggested in the haploid Old blush genome sequence and possibly contributes to its CF behavior $^{10}$, and some continuously flowering rose plants have no Copia insertion in their $K S N$ gene ${ }^{11,12}$; thus, the mutation of $K S N$ may not be the only reason for the rose CF trait.

In Arabidopsis, TFL1 maintains the indeterminate growth of the inflorescence meristem by inhibiting AP1 and $L F Y$ expression ${ }^{13,14}$. In $t f l 1$ mutants, indeterminate meristems are rapidly converted into determinate meristems, which produce terminal flowers ${ }^{13,15,16}$. Functional characterization of FvTFL1 has confirmed its role as a floral repressor that causes OF: mutations in this gene lead to CF in strawberry ${ }^{9,17}$. In perennial woody plant species, silencing the TFL1 homolog shortens the juvenile period and causes continuous flowering. For example, silencing MdTFL1 in apple (Malus domestica) reduces the juvenile period from 5 years to several months ${ }^{18,19}$, whereas silencing PcTFL1 in pear (Pyrus communis L.) accelerates flowering by $1-8$ months ${ }^{20}$. In Populus spp., silencing of the TFL1 orthologs PopCEN1/PopCEN2 hastens the first onset of flowering, maintains the indeterminate growth of axillary meristems and accelerates bud dormancy release upon chilling ${ }^{21}$. In perennial Arabis alpine plants, AaTFL1 regulates the duration of agedependent vernalization required for $A a L F Y$ expression and sets a threshold for flowering ${ }^{22}$. Therefore, TFL1 $(K S N)$ is functionally conserved as a flowering repressor.

Purple branch is a rare continuously flowering variety of $R$. rugosa that is cultivated widely in China. $R$. rugosa
Purple branch provides raw materials for the production of essential oils, jams, teas, pies, beverages, and other derivatives, integrating the primary, secondary and tertiary industries. To date, the origin of Purple branch remains unclear, but it is generally considered to have derived from an interspecific cross between $R$. rugosa Plena and Rosa davurica ${ }^{23,24}$. Intriguingly, its presumptive parents are OF rose species (Fig. 1); thus, its CF behavior remains unexplained. Elucidating the molecular mechanism of the CF trait in Purple branch will provide a theoretical basis for breeding new varieties with different flowering phenotypes.

In this paper, we demonstrate that the continuously flowering $R$. rugosa Purple branch is heterozygous for $K S N$ : it contains one normal transcribed wild-type allele and another transcriptionally blocked allele with a 9-kb Copia insertion, identical to that in $R$. chinensis Old blush. We show that the CF behavior of Purple branch is associated with reduced $K S N$ expression. This reduction is due to the halved dose of the wild-type $K S N$ allele and is linked to promoter hypermethylation and histone modification at the KSN locus. We thus present a novel mechanism for the production of the CF habit of rose plants.

\section{Materials and methods}

\section{Plant materials and growth conditions}

$R$. rugosa Purple branch, $R$. rugosa Plena, $R$. davurica and $R$. chinensis Old blush were grown in the rose resource nursery of Nanjing Agricultural University (Nanjing, China) under natural conditions.

Arabidopsis thaliana wild-type (Columbia), tfl1-14 mutant and transgenic lines were grown in a grown chamber under controlled conditions $\left(22^{\circ} \mathrm{C}, 40 \%\right.$ relative humidity, and $180 \mu \mathrm{mol} \mathrm{m}^{-2} \mathrm{~s}^{-1}$ of photosynthetically active radiation) under long-day conditions (16 h of light/ $8 \mathrm{~h}$ of darkness). 


\section{Gene cloning and expression analysis}

Genomic DNA from Purple branch, Plena, $R$. davurica and Old blush leaves was isolated by the CTAB method ${ }^{25}$. The different fragments of the KSN gene were isolated by PCR using the primers listed in Table S1. For expression analysis, shoot apices of field-grown Purple branch, Plena and $R$. davurica were collected on 29 March, 11 April and 30 September 2019, flash frozen in liquid nitrogen, and then stored at $-80^{\circ} \mathrm{C}$. Total RNA was extracted using a Vazyme FastPure Plant Total RNA Isolation Kit (Polysaccharides \& Polyphenolics rich, Vazyme Biotech, Nanjing, China). One microgram of high-quality total RNA was reverse transcribed using TransScript One-Step gDNA Removal and cDNA Synthesis SuperMix (Transgen Biotech, Beijing, China) according to the manufacturers' instructions. The cDNA was amplified via RT-PCR, and quantitative reversetranscription PCR (qRT-PCR) of KSN and FT was carried out with ABI QuantStudio 5 instrument (ABI Life Technologies, Carlsbad, USA) using the primers listed in Table S1; RcGAPDH was used as an internal reference ${ }^{26}$. Each experiment was conducted for three biological replicates, with three technical repeats each.

\section{Methylation-specific PCR (MSP)}

Methylation of the $K S N$ promoter was studied using MSP. Less than $500 \mathrm{ng}$ of genomic DNA was treated with sodium bisulfite using an EZ DNA Methylation Kit (Zymo Research, Irvine, USA). The treated DNA was subsequently used as a template for PCR. Approximately $1 \mathrm{~kb}$ of the KSN promoter region was amplified using two pairs of primers (Table S1) that were designed via Methyl Primer Express v1.0. The total volume of the PCR was $25 \mu \mathrm{l}$, and PCR was performed using TaKaRa EpiTaq HS (for bisulfite-treated DNA) according to the following protocol: 35 cycles of $98^{\circ} \mathrm{C}$ for $10 \mathrm{~s}, 55-60^{\circ} \mathrm{C}$ for $30 \mathrm{~s}$, and $72^{\circ} \mathrm{C}$ for $30 \mathrm{~s}$, followed by $72{ }^{\circ} \mathrm{C}$ for $10 \mathrm{~min}$. The resulting PCR fragments were ligated into a pMD-18-T vector (TaKaRa, Dalian, China), which was then transformed into Escherichia coli (Trans-T1) (Transgen Biotech, Beijing, China), and 10 clones per sample were sequenced using a bioanalyzer. Each experiment was performed for three biological repeats. The methylation percentages of cytosine $(\mathrm{C})$ in $\mathrm{CG}, \mathrm{CHH}$ and $\mathrm{CHG}(\mathrm{H}=\mathrm{A}, \mathrm{C}$ or $\mathrm{T})$ were analyzed using online software (http://katahdin.mssm.edu/ kismeth/revpage.pl), and the running parameters were as follows: minimum fraction of positive matches, 0.8 ; minimum fraction of length, 0.5 .

\section{Chromatin immunoprecipitation PCR (ChIP-PCR)}

ChIP-PCR was performed as described previously ${ }^{27}$, with slight modifications. Shoot apices of field-grown Purple branch and Plena were collected in September 2020 and ground in liquid nitrogen in M1 buffer $(10 \mathrm{mM}$ phosphate buffer $[\mathrm{pH} \quad 7.0], \quad 0.1 \mathrm{M} \mathrm{NaCl}, \quad 10 \mathrm{mM}$ mercaptoethanol, $1 \mathrm{M}$ hexylene glycol, $1 \times$ protease inhibitor cocktail [Roche], 5\% PVP, and $1 \mathrm{mM}$ PMSF). The suspension was filtered through four layers of Miracloth, after which the filtrate was centrifuged at $12,000 \mathrm{rpm}$ for $10 \mathrm{~min}$. The pelleted chromatin was washed thrice with M2 buffer (M1 buffer plus $10 \mathrm{mM} \mathrm{MgCl} 2$ and $0.5 \%$ Triton $\mathrm{X}-100)$ and once with $\mathrm{M} 3$ buffer $(10 \mathrm{mM}$ phosphate buffer [pH 7.0], $0.1 \mathrm{M} \mathrm{NaCl}, 10 \mathrm{mM}$ mercaptoethanol, $1 \times$ protease inhibitor cocktail [Roche], and $1 \mathrm{mM}$ PMSF). The chromatin was resuspended in nuclear lysis buffer and sonicated to generate DNA fragments of approximately $500 \mathrm{bp}$. The lysate was precleared by incubation together with $50 \mu \mathrm{l}$ of protein-A agarose beads/salmon sperm DNA (Millipore, Billerica, USA) for $1 \mathrm{~h}$. It was then incubated together with IgG, anti-H3K4me3, antiH3K9me3, and anti-H3K27me2 antibodies (Abcam, Cambridge, UK) overnight. The bound DNA fragments were recovered and purified using columns from a plasmid extraction kit (Qiagen, Hilden, Germany) according to the manufacturer's instructions. Quantitative real-time PCR was subsequently performed using the bound and input DNA as templates in conjunction with the primers listed in Table S1.

\section{Transient transformation system}

The coding regions of MET1, CMT3, JMJ12, and SUVR5 of $R$. rugosa Purple branch were cloned using the primers listed in Table S1 and then sequenced. The resulting sequences were used for phylogenetic analysis with Arabidopsis thaliana to validate their reliabilities and then submitted to the NCBI database.

To silence specific genes using RNAi, the conserved fragments of MET1 (MW012566), CMT3 (MW012565), JMJ12 (MW012568) and SUVR5 (MW012567) were amplified from cDNA of Purple branch using specific primers (Supplemental Table S1). The 300-400 bp amplicons were then subcloned into a pENTR-D-TOPO vector (Invitrogen, Carlsbad, USA). A pFAST-R03 binary vector (http://www.psb.ugent.be/) was used in the subsequent LR recombination to generate RNAi plasmids.

To analyze the RNAi effects, young shoots of Purple branch were transiently transformed as previously described $^{28}$. Briefly, young shoots were placed into a 50$\mathrm{mL}$ Agrobacterium solution carrying the gene fragments of interest and vacuum infiltrated for $5 \mathrm{~min}$, and an Agrobacterium solution carrying the empty vector served as a control. After being released from the vacuum, the shoots were washed with deionized water, and the leaves were collected for RNA extraction and q-PCR analysis after 3 days.

\section{Statistical analyses}

Statistical analysis of the data was performed via SPSS 17 statistical software. Two groups of data were compared 
using Kolmogorov-Smirnov (KS) tests (" $P<0.05$; **P $P<$ 0.01). Multiple groups of data were compared using the Kruskal-Wallis (KW) test, with $P<0.05$ considered significant.

\section{Results}

Purple branch contains two KSN alleles

We cloned the $K S N$ promoter and coding region from continuously flowering Purple branch, OF Plena, and $R$. davurica using the same primers that were previously used to amplify this region in $R$. chinensis Old blush ${ }^{29}$. We used the primer pair F1/R1 to amplify the promoter fragment and the primer pair F1/R2 to clone the fulllength $K S N$ gene, including both the promoter and coding areas (Fig. 2a). Based on the results of electrophoresis analysis of the PCR products on agarose gels, we obtained two bands from the Purple branch, with primers F1/R1 or F1/R2, and only one band of KSN from Plena and $R$. davurica (Fig. 2b, d).

Subsequent sequence alignment showed that the sequence of one of the KSN alleles from Purple branch was almost the same as that from Plena, except for a few
SNPs and a $44 \mathrm{bp}$ insertion in the second intron. The other allele was similar to that from CF $R$. chinensis Old blush and contained a Copia retrotransposon insertion in the second intron (Figs. 2c, e and S1). These results indicate that the diploid Purple branch is heterozygous for $K S N$ (KSN-Copia/KSN-Wt-Pb) (Fig. 2f). Because neither allele was similar to that of $R$. davurica, our work contradicts the notion that Purple branch was derived from an interspecific cross between $R$. rugosa Plena and $R$. davurica.

\section{$K S N$ expression is lower in Purple branch than in Plena and \\ R. davurica}

Because one of the $K S N$ alleles contains a $9 \mathrm{~kb}$ Copia insertion in the second intron in Purple branch, we tested whether the other allele contains a $44 \mathrm{bp}$ insertion in the second intron that was transcribed normally. We cloned the coding regions of $K S N$ from the cDNA of Purple branch, Plena, and $R$. davurica. We obtained a single band from the three different genotypes and sequenced the amplicons (Fig. 3a). Purple branch was identical to Plena (Fig. 3d, e) and contained a few bases that were different

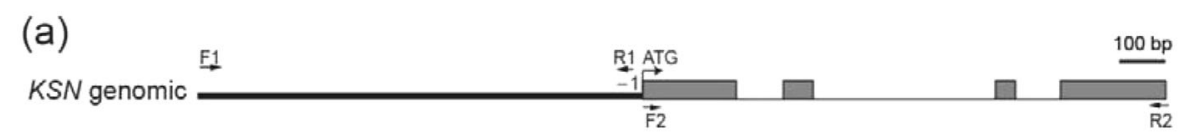

(b)

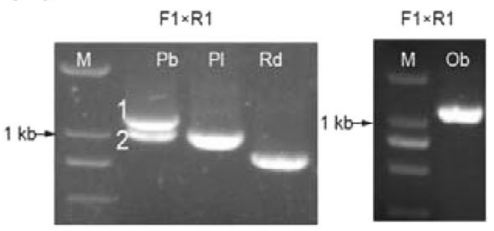

(d)

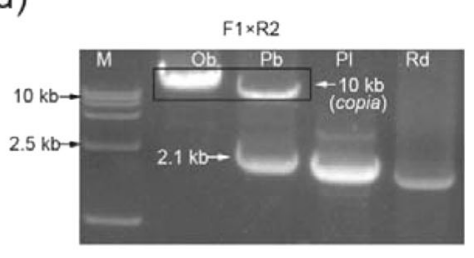

(c)

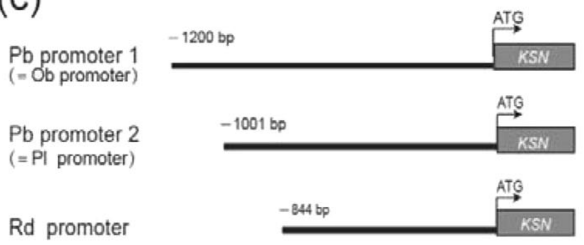

(e)

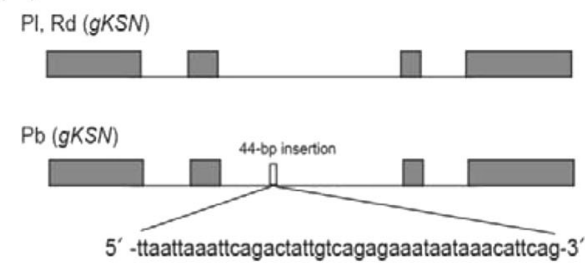

(f)

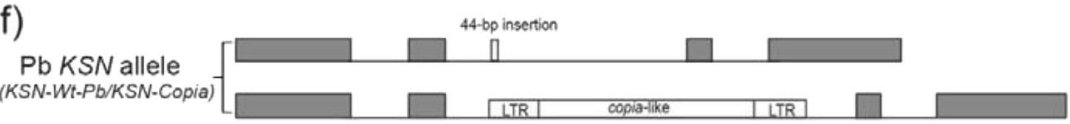

Fig. 2 Heterozygous KSN-Copia/KSN-Wt-Pb in continuously flowering Purple branch. a Schematic representation of the KSN gene in Rosaceae. The promoter is represented by a thick black line, exons are represented by gray boxes, introns are represented by thin black lines, and primers are indicated with arrows. b PCR products separated on an agarose gel by electrophoresis using primers F1/R1 in Purple branch (Pb), Plena (PI), $R$. davurica (Rd) and $R$. chinensis Old blush (Ob). Only one band is amplified in OF PI and Rd, whereas there are two bands (1 and 2) in CF Pb. c Sequence alignment indicating that $\mathrm{Pb}$ promoter 1 is identical to the KSN promoter in Ob and that Pb promoter 2 is identical to the KSN promoter in PI. $\mathbf{d}$ PCR amplification of KSN using primers F1/R2 in Pb, Pl, Rd and Ob. e Sequence alignment of the $2.1 \mathrm{~kb}$ product in $\mathrm{Pb}$ : the second intron contains a $44 \mathrm{bp}$ insertion compared with the second intron of $\mathrm{Pl}$ and $\mathrm{Rd}$. $\mathbf{f}$ Schematic representation of the heterozygous KSN-Copia/KSN-Wt-Pb in CF Pb 
(a)

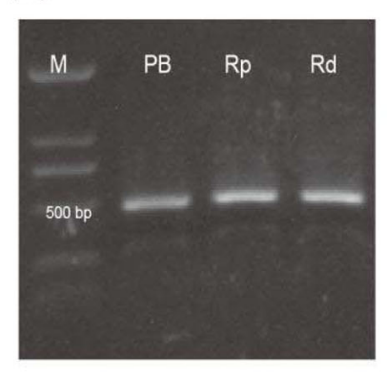

(b)

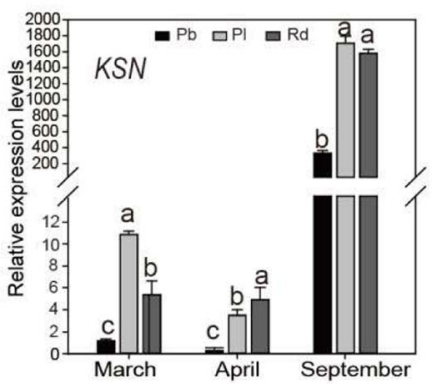

(c)

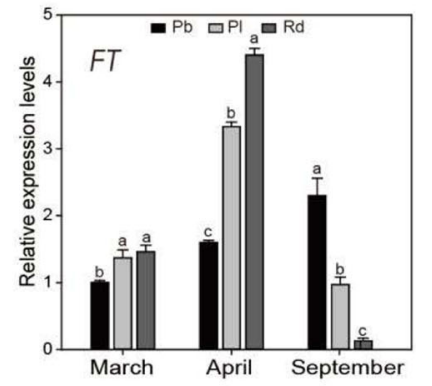

(d)

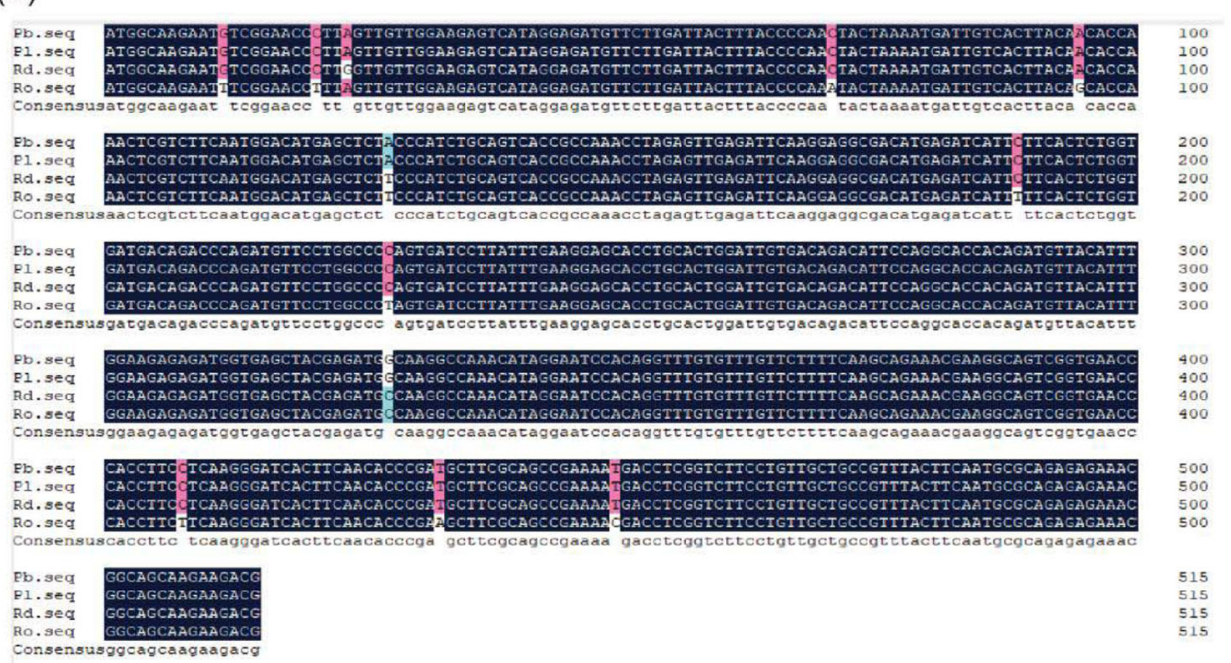

(e)
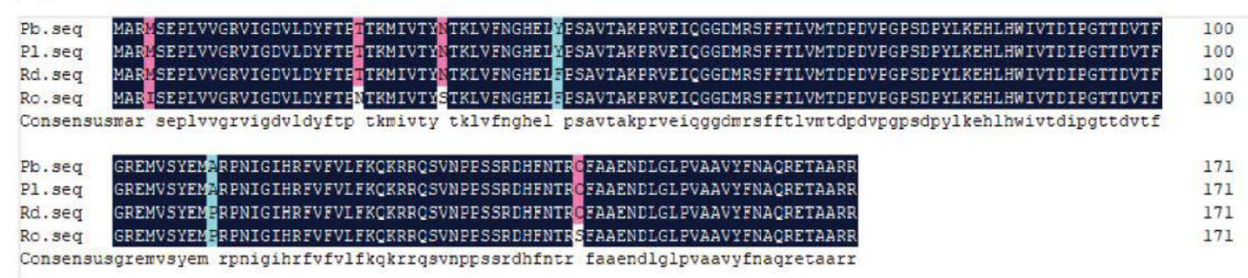

Fig. 3 Expression analysis of $K S N$ from Purple branch, Plena and $\boldsymbol{R}$. davurica. a PCR amplification of KSN using primers F2/R2 from CDNA of Purple branch (Pb), Plena (PI) and R. davurica (Rd). b, c Expression levels of KSN and FT from Pb, Pl, and Rd in March, April, and September. d, e Show the nucleotide sequence and amino acid sequence alignments of KSN genes from $\mathrm{Pb}, \mathrm{Pl}, \mathrm{Rd}$, and $R$. wichurana (Ro). The letters above the bars indicate significant differences, as determined by the KW test $(P<0.05)$

from those of $R$. davurica and $R$. chinensis Old blush. Thus, continuously flowering Purple branch carrying a wild-type $K S N$ allele can be transcribed normally without interference by the $44 \mathrm{bp}$ insertion.

To further compare the expression levels of $K S N$ among Purple branch, Plena, and $R$. davurica and determine the link between $K S N$ and flowering modes, we conducted qRT-PCR on samples collected in March (before blooming), April (during blooming), and September (continuously flowering Purple branch blooms, but OF $R$. rugosa Plena and $R$. davurica do not bloom) from all three genotypes. KSN expression was inhibited before flowering, maintained at relatively low levels during flowering, but obviously increased after flowering in September not only in OF $R$. rugosa Plena and $R$. davurica but also in continuously flowering Purple branch (Fig. 3b). However, the KSN expression in continuously flowering Purple branch being dramatically lower than that in OF Plena and $R$. davurica at all three time points may be caused by halved doses, leading to a CF habit. We also analyzed FT expression at the same time points. The expression levels of $F T$ in Purple branch, Plena, and $R$. davurica were all higher in April than in March, and the levels still increased in September in continuously flowering Purple 
(a)

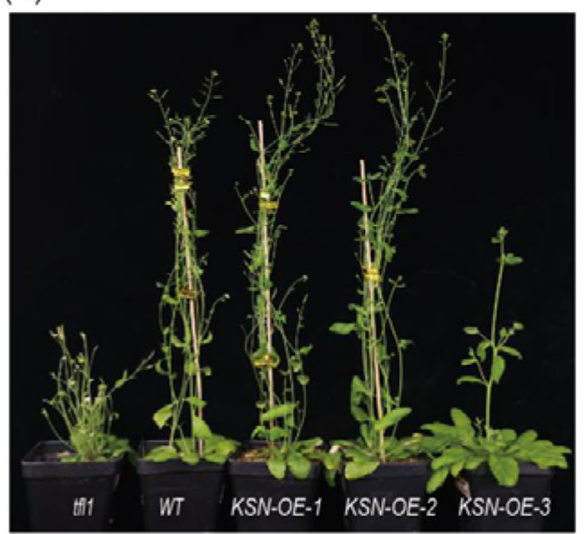

(b)

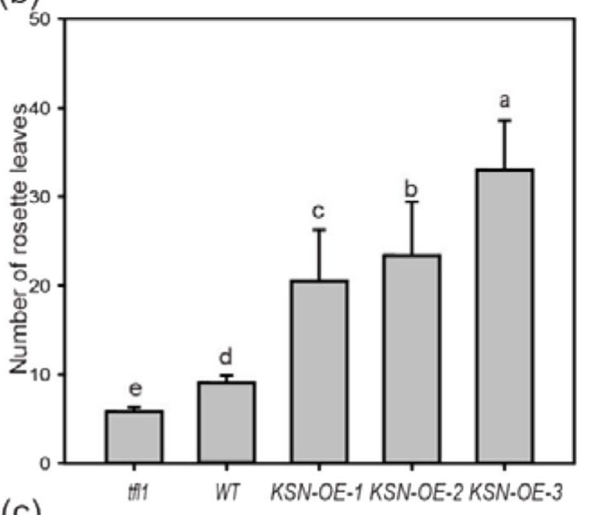

(c)

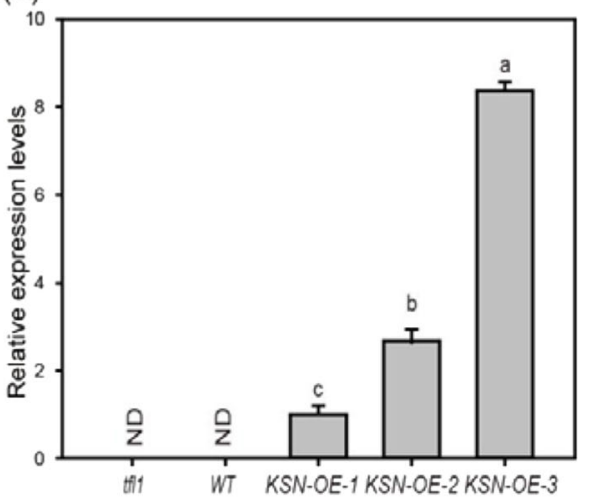

Fig. 4 Ectopic expression of KSN from continuously flowering Purple branch rescues the early flowering phenotype of the Arabidopsis tfl1 mutant. a Phenotype of Arabidopsis wild type (WT); tfl1-14 mutants; and the KSN heterologous expression lines KSN-OE-1, $K S N-O E-2$, and KSN-OE-3. b Rosette leaves of the above genotypes at flowering. c Expression levels of KSN in the above genotypes. The error bars indicate the standard deviations $(n=3)$. The different letters above the bars indicate significant differences, as determined by the KW test $(P<0.05)$

branch but declined significantly in OF $R$. rugosa Plena and $R$. davurica (Fig. 3c). Therefore, KSN expression was negatively associated with the flowering transition in the spring, and the flowering of Purple branch in autumn may be due to the integrative effects of both $K S N$ and $F T$.

\section{$K S N$ from Purple branch rescues the early-flowering $t f 1$ mutant phenotype}

To determine the functional conservation of the $K S N$ transcript from Purple branch, we expressed this gene under the constitutive $35 \mathrm{~S}$ promoter in the Arabidopsis tfl1 mutant background. We then examined the flowering phenotype and gene expression levels in the transgenic plants.

The heterologous expression of KSN from Purple branch rescued the early flowering and determinate growth of the inflorescence of $t f l 1$ mutants (Fig. 4a, b). The $t f l 1-14$ mutant produced 5.8 rosette leaves in contrast to the 9 rosette leaves produced by wild-type Arabidopsis at flowering. The flowering of the $K S N-O E-1$ and $K S N-$ $O E-2$ lines was delayed to occurring when there were more than 20 rosette leaves. This delay was associated with increased $K S N$ mRNA levels. Seedlings of the $K S N$ $O E-3$ line, with relatively high $K S N$ expression levels, were more delayed and produced more than 30 rosette leaves before flowering (Fig. 4b, c).

In addition, some transgenic seedlings produced leaflike floral organs that generated no seeds. These results corroborated those from studies of overexpression of TFL1 from Vitis vinifera, Lotus japonicus and Rosa wichurana in Arabidopsis ${ }^{30-32}$. Thus, the KSN of Purple branch is the functional homolog of TFL1 in Arabidopsis and other species and acts as a floral repressor.

\section{The KSN promoter in Purple branch is hypermethylated}

We hypothesized that the heterozygosity-induced dose decrease may not be the only reason for the inhibition of $K S N$. The $9 \mathrm{~kb}$ insertion may alter the chromatin structure and induce epigenetic suppression or activation. Using MSP, we tested the methylation status of CpG islands in the promoter of the normally transcribed $K S N$ allele in Purple branch. The B fragment ( -984 to $-502 \mathrm{bp}$ ) rather than the A fragment ( -501 to $-92 \mathrm{bp}$ ) was identified as the CpG island for DNA methylation (Fig. 5a, b). The total percentages of the three types of methylation (CG/CHG/ $\mathrm{CHH}$ ) within the $\mathrm{B}$ fragment were slightly higher in continuously flowering Purple branch than in OF Plena at all three time points (Fig. 5d), while it was higher only in April within the A fragment in continuously flowering Purple branch (Fig. S2a and S2c). Of the three types of methylation, CG and CHG methylation were most common (Figs. 5c and S2b). The methylation intensity within the promoter was negatively associated with the corresponding level of the KSN transcript presented in Fig. 3. Thus, hypermethylation may have partially contributed to the lower expression of KSN in Purple branch. 


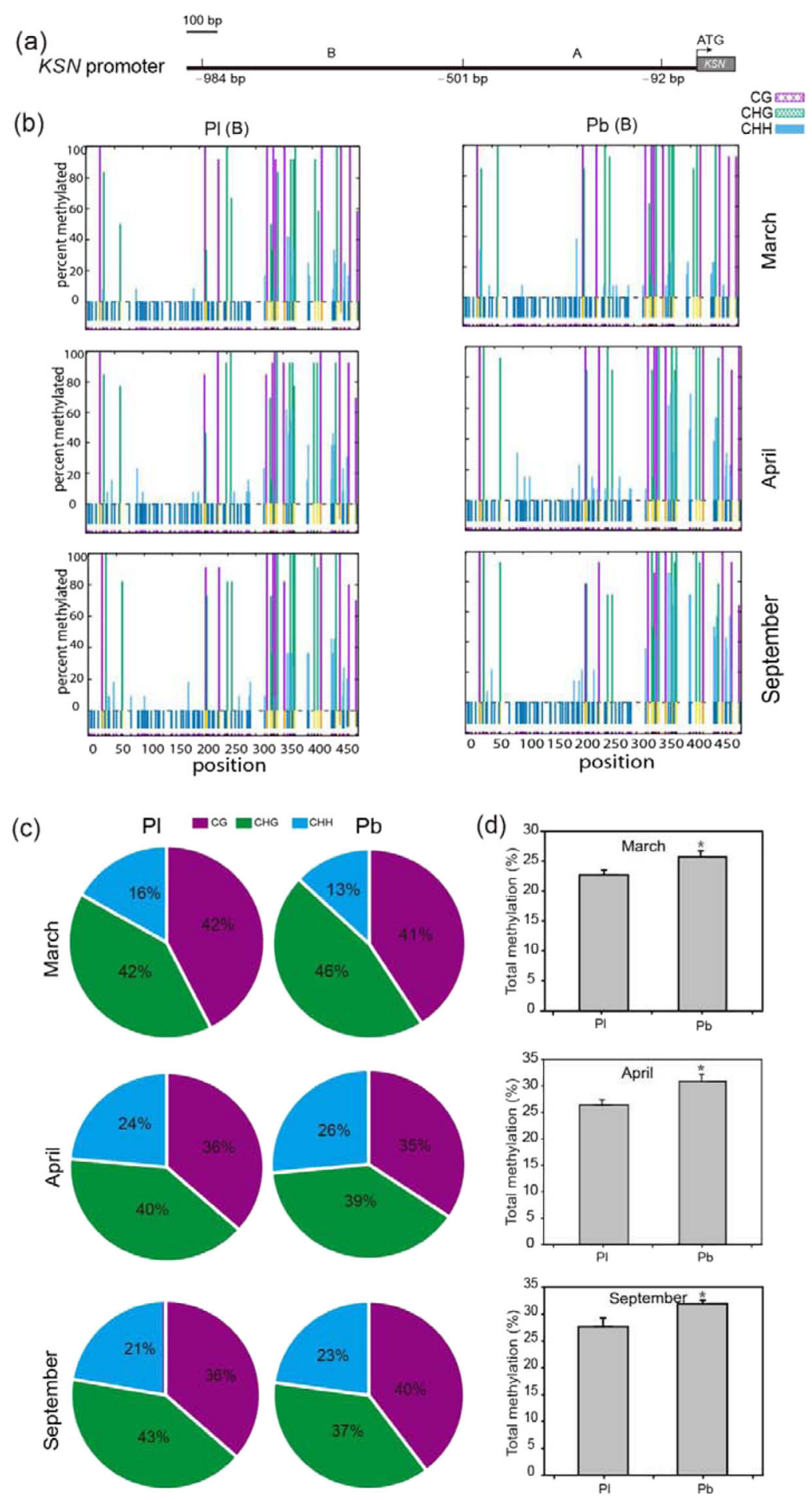

Fig. 5 Region B methylation status of the $K S N$ promoter in Purple branch and Plena. a Schematic representation of fragment B (-984 to -501 bp) and fragment $\mathrm{A}$ ( -501 to $-92 \mathrm{bp}$ ) of the KSN promoter. $\mathbf{b}$ The methylation status of $\mathrm{CG} / \mathrm{CHG} / \mathrm{CHH}$ sites in region B of KSN promoters from Purple branch $(\mathrm{Pb})$ and Plena (PI) in March, April, and September. The colored lines above the X-axis show the percentage of methylation at individual cytosine sites. The short bars at the bottom of the graph show the positions of the cytosines. $\mathbf{c}$ The pie chart shows the percentage of region B with three types of methylation at different stages in $\mathrm{Pb}$ and $\mathrm{Pl}$. $\mathbf{d}$ Comparisons of the total methylation percentage of region $\mathrm{B}$ in $\mathrm{Pb}$ and $\mathrm{Pl}$ according to Kismeth software (http://katahdin.mssm.edu/kismeth/revpage.pl). The error bars indicate the standard deviations $(n=3)$. The asterisks above the bars indicate significant differences, as determined by KS tests $\left({ }^{*} P<0.05\right)$ 
(a)

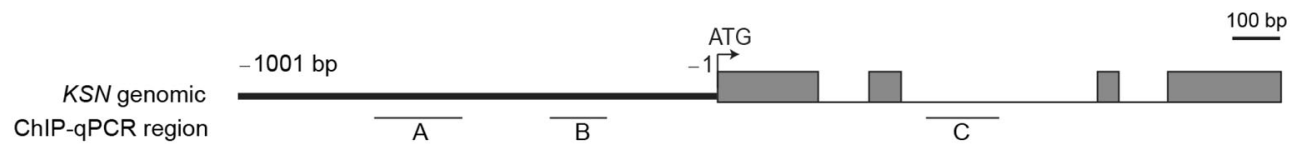

(b)
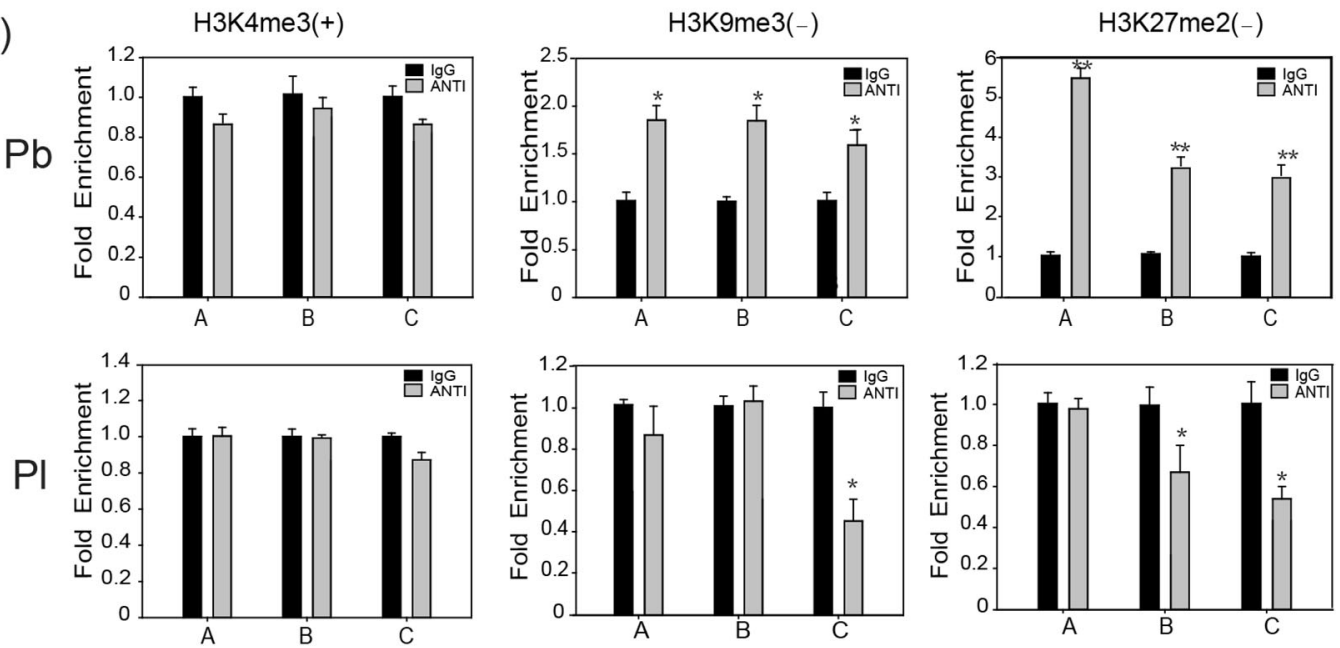

Fig. 6 Histone methylation of the $K S N$ locus in Purple branch and Plena. a Schematic representation showing ChIP-qPCR regions A (-638 to -496 bp), B (-387 to -240 bp) and C (494 to 595 bp), marked by black bars below the KSN genomic diagram. b Enrichment of H3K4me3, H3K9me3 and H3K27me2 in different regions of KSN from Purple branch (Pb) and Plena (PI). IgG was used as a negative control, and ANTI indicates the corresponding antibodies. The error bars indicate the standard deviations $(n=3)$. The asterisks above the bars indicate significant differences, as determined by KS tests $\left({ }^{*} P<0.05 ;{ }^{*} P<0.01\right)$

\section{Histone methylation of the KSN locus in Purple branch is modified}

To investigate how histone modification regulates $K S N$ expression, we collected shoot apices from fieldgrown Purple branch and Plena and analyzed KSN expression and the chromatin environment at the KSN locus in continuously flowering Purple branch and OF Plena via ChIP using H3K4me3-specific antibodies (to determine active loci) and H3K9me3- and H3K27me2specific antibodies (to determine silent loci). We designed specific primers throughout the promoter and the second intron regions (Fig. 6a and Table S1) and performed ChIP-qPCR. KSN expression was obviously lower in Purple branch than in Plena (Fig. S3), which is in line with the previous results shown in Fig. 3. The histone methylation marks H3K9me3 and H3K27me2 increased in continuously flowering Purple branch but remained unchanged or decreased in OF Plena. The H3K4me3 mark decreased in continuously flowering Purple branch but remained unchanged in OF Plena (Fig. 6b). Thus, the enhanced histone methylation at H3K27 and H3K9 and the reduced methylation at H3K4 may play important roles in maintaining the lower expression of the normal KSN allele in Purple branch, leading to its $\mathrm{CF}$ behavior.

\section{$K S N$ expression increases upon silencing MET1, CMT3, and SUVR5}

In Arabidopsis, DNA methylation is mainly performed by members of the C5-MTase families. MET1, which belongs to the METHYLTRANSFERASE (MET) family, maintains CG methylation. CMT3, which belongs to the CHROMOMETHYLTRANSFERASE (CMT) family, maintains CHG methylation ${ }^{33}$. The cmt 3 mutant shows a near-complete loss of $\mathrm{CpXpG}$ methylation, and met1 shows a marked reduction in $\mathrm{CpG}$ methylation; both types of methylation in turn cause gene silencing ${ }^{34,35}$. Histone modifications affect various changes in chromatin structure, leading to the promotion or suppression of gene expression ${ }^{36}$. JMJ12 (REF6), which belongs to the KDM4/JHDM3 group of the JmjC family ${ }^{37}$, encodes a histone H3 lysine 27 demethylase ${ }^{38}$, and silencing of RcJMJ12 induced late flowering in Rosa chinensis ${ }^{39}$. The SET family gene SUVR5 regulates flowering time through H3K9me2 and H3K27me3 and is independent of the vernalization pathway ${ }^{40-42}$.

To examine the epigenetic regulation of $K S N$ expression in Purple branch, we cloned the homologs of Arabidopsis MET1, CMT3, JMJ12, and SUVR5 from continuously flowering Purple branch and constructed phylogenetic trees together with their homologs in Arabidopsis to 
(a)

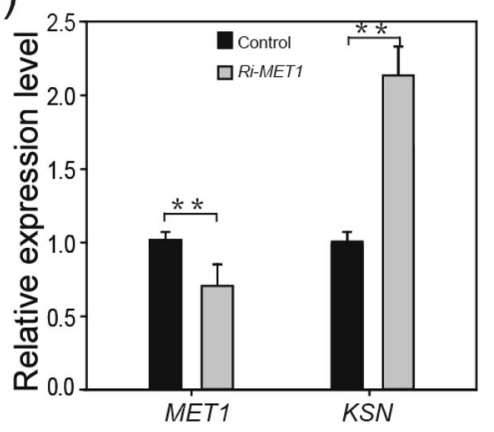

(c)

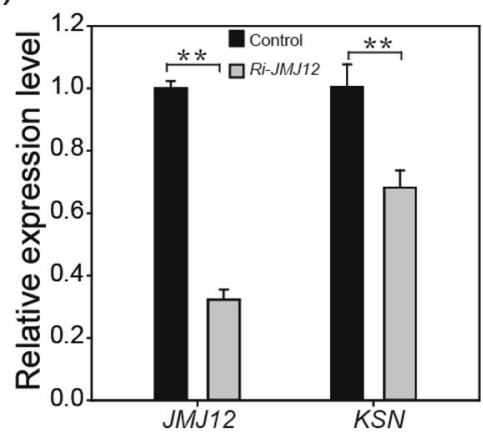

(b)

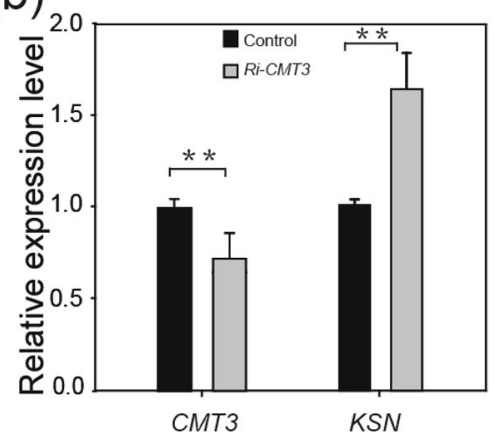

(d)

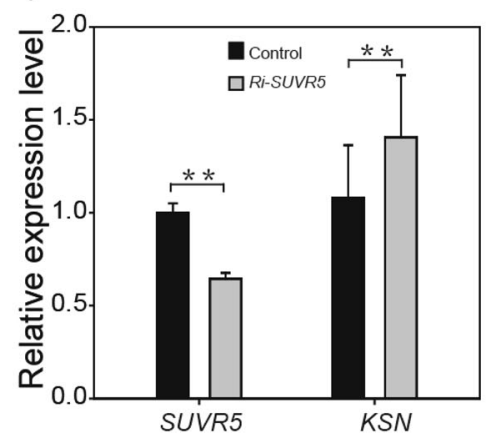

Fig. 7 Silencing MET1, CMT3, and SUVR5 increases KSN expression in Purple branch. a, b Expression levels of MET1, CMT3, and KSN in Purple branch seedlings of Ri-MET1 and Ri-CMT3 lines. c, d Expression levels of JMJ12, SUVR5, and KSN in Purple branch seedlings of Ri-JMJ12 and Ri-SUVR5 lines. Gene expression was measured via qRT-PCR for three biological replicates, with three technical repeats each. The RcGADPH gene was used as internal reference. The asterisks above the bars indicate significant differences, as determined by KS tests ( ${ }^{* *} P<0.01$ )

verify their correctness before submitting the sequences to the NCBI database (Fig. S4). We then designed primers (listed in Supplemental Table S1) specific to their conserved regions and silenced the genes in young rose shoots using a transient transformation system described previously $^{28}$. The subsequent qRT-PCR results showed that silencing MET1, CMT3, JMJ12, and SUVR5 significantly altered the DNA methylation/histone acetylation status at the KSN locus (Figs. S5 and S6), which was followed by a significant increase in $K S N$ transcription in Ri-MET1, Ri-CMT3 and Ri-SUVR5 seedlings compared with a decrease in KSN expression in Ri-JMJ12 seedlings of continuously flowering Purple branch (Fig. 7). Thus, DNA methylation and histone methylation are potentially partially responsible for the repression of KSN in Purple branch.

\section{Discussion}

Previous studies have genotyped $K S N$ in a wide range of rose cultivars and established that some Damask-related roses (e.g., Damask, Moss, Hybrid musk, and Bourbon roses) and Asian roses (e.g., Hybrid rugosa and Hybrid bracteate roses) were CF types but contained no mutated alleles of $K S N$ from $R$. chinensis ${ }^{12}$. Similarly, it was also found that continuously flowering $R$. rugosa Hamanasu from Japan had only a wild $K S N$ allele without any insertion or obvious mutations ${ }^{11}$. Therefore, the CF behavior of rose plants may not be simply explained by the mutation or insertion of the $K S N$ gene. Interestingly, the present results clearly showed that the KSN allele with the Copia insertion from $R$. chinensis was substantial in $R$. rugosa Purple branch (Figs. 2 and 3), which is heterozygous (KSN-Copia/KSN-Wt-Pb) at the KSN locus. The wild-type $K S N$ allele was proven to be functional via heterologous expression in Arabidopsis (Fig. 4). This discrepancy may be caused by different experimental materials or methods, as there are different cultivars and varieties of $R$. rugosa from different origins. Additionally, the $K S N$ allele carrying the $9 \mathrm{~kb}$ insertion was not easily amplified with the normal DNA polymerase and general PCR protocol.

Next, KSN expression was suppressed during the floral initiation period in early spring (April) and dramatically upregulated in the shoot apices after flower initiation in all three species, although $K S N$ expression was still much lower in continuously flowering Purple branch than OF Plena and $R$. davurica in September (Fig. 3b). In contrast, expression of the floral activator FT significantly increased 
in April in all three species, decreased in OF Plena and $R$. davurica but continued climbing in continuously flowering Purple branch (Fig. 3c). Consequently, continuously flowering Purple branch exhibited high expression of both FT and KSN in September. Plant flowering is precisely determined by the equilibrium between floral promoters and repressors. FT, a member of the phosphatidylethanolamine-binding protein (PEBP) family, is a key florigen that integrates the internal and external signals for floral transition ${ }^{434}$. In the shoot apical meristem (SAM), FT interacts with FD to activate floral identity genes such as $A P 1$ and $L F Y$ and initiates floral bud formation ${ }^{45,46}$. Like the flowering promoter FT, TFL1 is also classified as a PEBP member ${ }^{47}$ but with a function opposite that of FT, acting as a floral repressor. TFL1 interacts with FD to form a TFL1-FD complex, opposing the function of the FT-FD complex in regulating downstream gene expression ${ }^{48}$. Thus, the floral activator FT may antagonize or suppress the repressor KSN in continuously flowering Purple branch, enabling its flowering in autumn.

Nevertheless, the overall expression of $K S N$ was clearly linked to the alternation of vegetative/reproductive stages of rose plants in the present paper and in six other nonrecurrent flowering species and nine recurrent flowering cultivars $^{49}$. Furthermore, the expression level of $K S N$ in continuously flowering Purple branch was obviously lower than that in OF Plena and $R$. davurica at all three time points, which was convincingly associated with the CF trait of Purple branch. Although other factors may be involved in controlling the $\mathrm{CF}$ trait, the roles of reduced $K S N$ expression should be predominant and can be mainly attributed to the decreasing dose associated with the heterozygosity of KSN loci in Purple branch (Figs. 2 and 3).

All plants have various transposons (TEs) that can disrupt local gene structure, affect the expression of nearby genes, and induce chromosomal instability. Most TEs are silenced and immobilized under normal conditions. DNA methylation and histone methylation are reversible epigenetic modifications that control transposon activity ${ }^{50-52}$. The $9 \mathrm{~kb}$ Copia-like retrotransposon insertion in $R$. chinensis Old blush not only blocks transcription of the host $K S N$ allele but also results in a large rearrangement at the CF locus, leading to the complete deletion of the other $K S N$ allele ${ }^{11}$. Therefore, the diploid Old blush is hemizygous: RoKSN $N^{\text {Copia }} / R o K S N^{\text {null }}$. This allowed us to test the DNA and histone methylation status of the $K S N$ locus. We observed hypermethylation of the $K S N$ promoter and enhanced methylation levels of H3K9 and H3K27 at the KSN locus in continuously flowering Purple branch compared with OF varieties without Copia insertions (Figs. 5 and 6). These results implied that the presence of Copialike retrotransposon insertion may potentially affect the epigenetic status of the KSN locus in continuously flowering Purple branch. Further silencing the DNA methyltransferase genes MET1 and CMT3 and histone methyltransferase gene SUVR5 in young shoots significantly increased the expression of $K S N$ in the Purple branch (Fig. 7), highlighting the importance of epigenetic modification in $K S N$ expression. Taken together, these data suggested that the reduction in $K S N$ expression may be associated with epigenetic modifications, except for the halved dose in continuously flowering Purple branch.

In conclusion, the $R$. rugosa Purple branch was found to be heterozygous at $K S N$, and its CF trait was associated with the lower expression of KSN. The reduction in KSN was caused by the halved dose and was also associated with hypermethylation of the promoter region and histone modification at the KSN locus following the $9 \mathrm{~kb}$ Copia insertion in the other allele. This suggests a novel mechanism for the production of the CF habit in rose plants.

\section{Acknowledgements \\ This work was supported by the National Key R\&D Program of China (2019YFD1000400), the NSFC (31972449) and the NSFC-XINJIANG joint foundation (U1803102), which provided grants to Changquan Wang; the NSFC (31801890), which provided a grant to Jinyi Liu; and the Postgraduate Research \& Practice Innovation Program of Jiangsu Province, which provided a grant to Mengjuan Bai.}

\section{Author contributions}

Mengjuan Bai and Jinyi Liu conceived and designed the experiments; Chunguo Fan, Yeqing Chen, Hui Chen, Jun Lu, and Jingjing Sun performed parts of the experiments; and Guogui Ning and Changquan Wang wrote the manuscript.

\section{Conflict of interest}

The authors declare that they have no conflict of interest.

Supplementary Information accompanies this paper at (https://doi.org/ 10.1038/s41438-021-00464-8).

Received: 24 July 2020 Revised: 10 November 2020 Accepted: 13 November 2020

Published online: 01 February 2021

\footnotetext{
References

1. Hurst, C. Notes on the origin and evolution of our garden roses. J. R. Hortic. Soc. 66, 282-289 (1941).

2. Martínez, M. C. \& Santiago, J. L. Narcea-an unknown, ancient cultivated rose variety from northern Spain. Hortic. Res. 7, 44 (2020).

3. Ng, T. B., He, J. S., Niu, S. M., Zhao, L. \& Liu, F. A gallic acid derivative and polysaccharides with antioxidative activity from rose (Rosa rugosa) flowers. J. Pharm. Pharm. 56, 537-545 (2004).

4. Ueyama, Y., Hashimoto, S., Nii, H. \& Furukawa, K. The essential oil from the flowers of Rosa rugosa Thunb. var. plena Regel. Flavour Frag. J. 5, 219-222 (2010).

5. Srikanth, A. \& Schmid, M. Regulation of flowering time: all roads lead to Rome. Cell Mol. Life. Sci. 68, 2013-2037 (2011).

6. Bendahmane, M., Dubois, A., Raymond, O. \& Bris, M. L. Genetics and genomics of flower initiation and development in roses. J. Exp. Bot. 64, 847-857 (2013).

7. Kurokura, T., Mimida, N., Battey, N. H. \& Hytönen, T. The regulation of seasonal flowering in the Rosaceae. J. Exp. Bot. 64, 4131-4141 (2013).

8. Randoux, M. et al. Gibberellins regulate the transcription of the continuous flowering regulator, RoKSN, a rose TFL1 homologue. J. Exp. Bot 63, 6543-6554 (2012).
} 
9. Iwata, $\mathrm{H}$. et al. The TFL1 homologue KSN is a regulator of continuous flowering in rose and strawberry. Plant J. 69, 116-125 (2012).

10. Saint-Oyant, L. H. et al. A high-quality genome sequence of Rosa chinensis to elucidate ornamental traits. Nat. Plants 4, 473-484 (2018).

11. Horibe, T., Yamada, K., Otagaki, S., Matsumoto, S. \& Kawamura, K. Molecular genetic studies on continuous-flowering roses that do not originate from Rosa Chinensis. Acta Hortic. 1064, 185-192 (2015).

12. Araou, E. Diversité génétique et origine de la remontée de floraison chez le rosier. Rapport de Stage, UMR GenHort, Centre INRA d'Angers-Nantes, France (2011).

13. Shannon, S. \& Meeks-Wagner, D. R. A mutation in the Arabidopsis TFL1 gene affects inflorescence meristem development. Plant Cell 3, 877-892 (1991).

14. Parcy, F., Bomblies, K. \& Weigel, D. Interaction of LEAFY, AGAMOUS and TERMINAL FLOWER1 in maintaining floral meristem identity in Arabidopsis. Development 129, 2519-2527 (2002).

15. Bradley, D., Ratcliffe, O., Vincent, C., Carpenter, R. \& Coen, E. Inflorescence commitment and architecture in Arabidopsis. Science 275, 80-83 (1997).

16. Alvarez, J., Guli, C. L., Yu, X. H. \& Smyth, D. R. TERMINAL FLOWER: a gene affecting inflorescence development in Arabidopsis thaliana. Plant J. 2 103-116 (2010)

17. Koskela, E. A. et al. Mutation in TERMINAL FLOWER1 reverses the photoperiodic requirement for flowering in the wild strawberry Fragaria vesca. Plant Physiol. 159, 1043-1054 (2012).

18. Flachowsky, H. et al. The MdTFL1 gene of apple (Malusx domestica Borkh.) reduces vegetative growth and generation time. Tree Physiol. 32, 1288-1301 (2012).

19. Naozumi, M. et al. Four TFL1/CEN-like genes on distinct linkage groups show different expression patterns to regulate vegetative and reproductive development in apple (Malus x domestica Borkh.). Plant Cell Physiol. 50, 394-412 (2009).

20. Freiman, A. et al. Development of a transgenic early flowering pear (Pyrus communis L.) genotype by RNAi silencing of PCTFL1-1 and PCTFL1-2. Planta 235, 1239-1251 (2012).

21. Mohamed, R. et al. Populus CEN/TFL1 regulates first onset of flowering, axillary meristem identity and dormancy release in Populus. Plant J. 4, 674-688 (2010).

22. Wang, R. et al. Aa TFL1 confers an age-dependent response to vernalization in perennial Arabis alpina. Plant Cell. 4, 1307-1321 (2011).

23. Sun, Fanxia. A new member of the rose family-Purple bruch. Rural Practical Science and Technology Information 1, 16 (2007).

24. Gang, Ding, Xianshui, Meng \& Guizhi, Dong Purple bruch and its bud variety. China Flower Bonsai 12, 14-15 (2007).

25. Xu, Q., Wen, X. \& Deng, X. A simple protocol for isolating genomic DNA from chestnut rose (Rosa roxburghii tratt) for RFLP and PCR analyses. Plant Mol. Biol. Rep. 22, 301-302 (2004).

26. Liu, J. et al. MIKC C-type MADS-box genes in Rosa chinensis: the remarkable expansion of $A B C D E$ model genes and their roles in floral organogenesis. Hortic. Res. 5, 1-15 (2018).

27. Liu, X. et al. AGAMOUS terminates floral stem cell maintenance in Arabidopsis by directly repressing WUSCHEL through recruitment of Polycomb Group proteins. Plant Cell 23, 3654-3670 (2011).

28. Lu, J., Bai, M., Ren, H., Liu, J. \& Wang, C. An efficient transient expression system for gene function analysis in rose. Plant Methods 13, 116 (2017).

29. Iwata, H., Gaston, A. L., Remay, A., Thouroude, T. \& Jeauffre, J. The TFL1 homologue $\mathrm{KSN}$ is a regulator of continuous flowering in rose and strawberry. Plant J. 69, 116-125 (2011)
30. Carmona, M. J., Calonje, M. \& Martínez-Zapater, J. M. The FT/TFL1 gene family in grapevine. Plant Mol. Biol. 63, 637-650 (2007).

31. Guo, X., Zhao, Z., Chen, J., Hu, X. \& Luo, D. A putative CENTRORADIALIS/ TERMINAL FLOWER 1-like gene, Ljcen1, plays a role in phase transition in Lotus japonicus. J. Plant Physiol. 163, 436-444 (2006).

32. Randoux, M. et al. RoKSN, a floral repressor, forms protein complexes with RoFD and RoFT to regulate vegetative and reproductive development in rose. N. Phytol. 202, 161-173 (2014).

33. Cao, D. et al. Genome-wide identification of cytosine-5 DNA methyltransferases and demethylases in Solanum lycopersicum. Gene 550, 230-237 (2014).

34. Lindroth, A. M. et al. Requirement of CHROMOMETHYLASE3 for maintenance of CpXpG methylation. Science 292, 2077-2080 (2001).

35. Cao, X. et al. Role of the DRM and CMT3 methyltransferases in RNA-directed DNA methylation. Curr. Biol. 13, 2212-2217 (2003).

36. Jenuwein, T. \& Allis, C. D. Translating the histone code. Science 293, 1074-1080 (2001).

37. Huang, Y., Chen, D., Liu, C., Shen, W. \& Ruan, Y. Evolution and conservation of JmjC domain proteins in the green lineage. Mol. Genet Genomics 291, 33-49 (2016).

38. Lu, F., Cui, X., Zhang, S., Jenuwein, T. \& Cao, X. Arabidopsis REF6 is a histone H3 lysine 27 demethylase. Nat. Genet. 43, 715-719 (2011).

39. Dong, Y., Lu, J., Liu, J., Jalal, A. \& Wang, C. Genome-wide identification and functional analysis of JmjC domain-containing genes in flower development of Rosa chinensis. Plant Mol. Biol. 102, 417-430 (2020).

40. Thorstensen, T., Grini, P. E. \& Aalen, R. B. SET domain proteins in plant development. Biochim. Biophys. Acta 1809, 407-420 (2011).

41. Krichevsky, A., Kozlovsky, S. V., Gutgarts, H. \& Citovsky, V. Arabidopsis corepressor complexes containing polyamine oxidase-like proteins and plant-specific histone methyltransferases. Plant Signal. Behav. 2, 174-177 (2007).

42. Krichevsky, A. et al. $\mathrm{C} 2 \mathrm{H} 2$ zinc finger-SET histone methyltransferase is a plantspecific chromatin modifier. Dev. Biol. 303, 259-269 (2007)

43. Lin, M. K. et al. FLOWERING LOCUS T protein may act as the long-distance florigenic signal in the cucurbits. Plant Cell 19, 1488-1506 (2007).

44. Tamaki, S., Matsuo, S., Wong, H. L., Yokoi, S. \& Shimamoto, K. Hd3a protein is a mobile flowering signal in rice. Science 316, 1033-1036 (2007).

45. Fornara, F., de Montaigu, A. \& Coupland, G. SnapShot: control of flowering in Arabidopsis. Cell 141, 550.e1-550.e2 (2010).

46. Srikanth, A. \& Schmid, M. Regulation of flowering time: all roads lead to Rome. Cell Mol. Life Sci. 68, 2013-2037 (2011).

47. Karlgren, A. et al. Evolution of the PEBP gene family in plants: functional diversification in seed plant evolution. Plant Physiol. 156, 1967-1977 (2011).

48. Hanano, S. \& Goto, K. Arabidopsis TERMINAL FLOWER1 is involved in the regulation of flowering time and inflorescence development through transcriptional repression. Plant Cell 3, 3172-3184 (2011).

49. Wang, L. N., Liu, Y. F., Zhang, Y. M., Fang, R. X. \& Liu, Q. L. The expression level of Rosa Terminal Flower 1 (RTFL1) is related with recurrent flowering in roses. Mol. Biol. Rep. 39, 3737-3746 (2011).

50. Lippman, Z. et al. Role of transposable elements in heterochromatin and epigenetic control. Nature 430, 471-476 (2004).

51. Mirouze, M. et al. Selective epigenetic control of retrotransposition in Arabidopsis. Nature 461, 427-430 (2009).

52. Tsukahara, S. et al. Bursts of retrotransposition reproduced in Arabidopsis Nature 461, 423-426 (2009). 